\title{
A VISÃO DE CUIDADORES NO CUIDADO DE IDOSOS DEPENDENTES INSTITUCIONALIZADOS
}

\author{
Camila Aparecida Pinheiro Landim Almeida ${ }^{1}$ \\ Lenilson Borges Santos² \\ Luana Maria da Conceição ${ }^{3}$ \\ Nélida Maria Silva ${ }^{4}$ \\ Herica Emília Felix de Carvalho ${ }^{5}$ \\ Francisca Cecília Viana Rocha ${ }^{6}$ \\ Eliana Campêlo Lago ${ }^{7}$ \\ Mônica Motta Lino ${ }^{8}$
}

\footnotetext{
1 Graduada em Enfermagem. Doutora em Ciências. Docente Titular do Centro Universitário UNINOVAFAPI, vinculada ao Programa de Mestrado Profissional em Saúde da Família. Teresina/PI, Brasil. E-mail: camila@uninovafapi.edu.br.

2 Graduado em Enfermagem pelo Centro Universitário UNINOVAFAPI. Teresina/PI, Brasil. E-mail: nil11_bs@hotmail.com.

3 Graduada em Enfermagem pelo Centro Universitário UNINOVAFAPI. Teresina/PI, Brasil. E-mail: luanamaria.gt@hotmail.com.

4 Graduada em Enfermagem pelo Centro Universitário UNINOVAFAPI. Teresina/PI, Brasil. E-mail: nelidamikaelly@hotmail.com.

5 Graduada em Enfermagem. Mestre em Enfermagem pela Universidade Federal do Piauí - UFPI. Teresina/PI, Brasil. E-mail: herica_emilly@hotmail.com.br.

6 Graduada em Enfermagem. Mestre em Enfermagem. Docente do Centro Universitário UNINOVAFAPI, vinculada ao Departamento de Enfermagem. Teresina/PI, Brasil. E-mail: fceciliavr@hotmail.com

7 Graduada em Enfermagem e em Odontologia. Doutora em Biotecnologia. Coordenadora do Programa de Mestrado Profissional em Saúde da Família do Centro Universitário UNINOVAFAPI. Teresina/Pl, Brasil. E-mail: eliana@uninovafapi.edu.br.

8 Graduada em Enfermagem. Doutora em Enfermagem. Professora Adjunta da Universidade Federal de Santa Catarina - UFSC, vinculada ao Departamento de Enfermagem. Florianópolis/SC, Brasil. E-mail: monicafloripa@hotmail.com.
} 
resumo

Este estudo tem como objetivo analisar a visão de cuidadores formais no cuidado de idosos dependentes institucionalizados. Realizou-se uma pesquisa qualitativa, desenvolvida em uma Instituição de Longa Permanência para Idosos, em Teresina, Piauí, Brasil. Participaram nove cuidadores que prestavam cuidados a idosos dependentes institucionalizados. Os dados foram coletados nos meses de dezembro/2014 a março/2015, por meio de entrevistas, utilizando um roteiro temático semiestruturado. As entrevistas gravadas foram transcritas e analisadas pela técnica do discurso do sujeito coletivo. Os resultados foram apresentados em três categorias temáticas e três tabelas, contendo as ideias centrais, os trechos selecionados dos discursos individuais, os participantes e a frequência. Na primeira categoria, a visão do cuidador do idoso dependente institucionalizado, foram encontradas três ideias centrais: satisfação no cuidado, complexidade do cuidado e relacionamento entre o idoso e o cuidador. $\mathrm{Na}$ segunda categoria, dificuldades do cuidador para cuidar de um idoso dependente institucionalizado, também foram geradas três ideias centrais: rotina de cuidados, fragilidades físicas e emocionais e deambulação noturna. Por fim, na terceira categoria, facilidades do cuidador para cuidar de um idoso dependente institucionalizado, foram encontradas duas ideias centrais: bom-humor e comunicação. Face aos resultados, recomenda-se que as Instituições de Longa Permanência dos Idosos dediquem maior atenção em atividades que promovam o bem-estar dos cuidadores, além de estratégias de prevenção contra as dificuldades ocasionadas pelas atividades inerentes à rotina de cuidados prestados aos idosos dependentes institucionalizados.

palavras-chave

Cuidadores. Idoso. Idoso dependente.

Frente aos sucessivos estudos desenvolvidos para a melhoria da qualidade de vida do idoso, analisar a visão de cuidadores no cuidado de idosos dependentes institucionalizados representa um desafio constante para a prática em saúde gerontológica. Isso ocorre devido ao crescimento acelerado 
da população idosa mundial, o que reflete no aumento da demanda por instituições de cuidado para idosos e cuidadores envolvidos.

O aumento da população idosa no mundo tem representado uma grande mudança na sociedade moderna (MORALES-VIVES; VIGIL-COLET, 2012). Em 2009, segundo os dados da Organização das Nações Unidas (ONU), existiam em torno de 865 milhões de idosos no mundo, correspondendo a 12,3\% de toda a população. Estima-se que até 2050 haverá 2,4 bilhões de idosos, ou seja, 26,2\% da população. Ainda em 2009, no cenário brasileiro, haviam cerca de 21 milhões de pessoas com mais de 60 anos de idade, o que correspondia a 11,3\% da população (ONU, 2009). Ainda de acordo com as estimativas, em 2050 haverá cerca de 50 milhões de idosos apenas no Brasil (ONU, 2012).

Nesse sentido, o fenômeno do envelhecimento populacional tem gerado preocupações para os profissionais de saúde, no que tange ao planejamento e implementação de cuidados voltados à melhoria da qualidade de vida de pessoas idosas (LISBOA; CHIANCA, 2012). Pesquisadores chineses discutem que uma adequada estrutura familiar em domicílio é essencial para o bem-estar e qualidade de vida do idoso (WANG; ZHAO, 2012).

Em função do aumento das proporções numéricas dos idosos e da longevidade da população, acrescidos das dificuldades socioeconômicas e culturais, do comprometimento da qualidade de vida e saúde dos idosos e familiares, da ausência do cuidador no domicílio e os conflitos familiares, surge a demanda por Instituições de Longa Permanência para Idosos (ILPI) (IBGE, 2014), expressão adotada pela Sociedade Brasileira de Geriatria e Gerontologia (SBGG) para ser usado em substituição por "asilo", termo fortemente marcado por preconceitos historicamente constituídos, correspondendo a Long Term Care Institution (RISSARDO et al., 2012).

Uma das grandes preocupações no contexto da institucionalização dos idosos é a função do cuidador como alguém que assume um papel essencial de auxiliar o idoso na realização das diversas tarefas cotidianas (BRASIL, 2012b). De acordo com o estudo de Saraiva (2011), o ato de cuidar implica que os cuidadores desenvolvam atenção nos aspectos instrumentais, de natureza física, técnica e prática, e expressivos, sobretudo os cuidados afetivos e relacionais. Dessa forma, o cuidar orienta-se para preencher as necessidades psicológicas, fisiológicas e sociais da pessoa dependente. A partir desse cuidar, o cuidador formal deve assumir uma visão holística dessas necessidades, como um ser biopsicossocial.

Pesquisa recente destaca que os cuidadores de idosos institucionalizados devem ser profissionais com uma formação especializada devido à variedade de conhecimentos exigidos pertinentes às habilidades e competências esperadas 
(SILVA et al., 2015). Compreendendo a inserção significativa dos cuidadores de idosos na sociedade, Loreiro et al. (2013) reforçam sobre a necessidade de capacitação para o cuidador formal de idosos dependentes, devido às demandas por cuidados cada vez mais complexos e constantes. No entanto, a presença de um profissional capacitado para esta atividade não é uma realidade frequente.

Somado aos aspectos relevantes relacionados à importância da formação do cuidador dos idosos institucionalizados, várias pesquisas têm sido desenvolvidas com o intuito de conhecer dados relativos ao bem-estar físico e emocional dos cuidadores de ILPI. Entretanto, tais estudos foram realizados, em sua maioria, com cuidadores informais (ANJOS et al., 2015; ANJOS et al., 2014; LINO et al., 2016).

Diante da problemática que envolve a temática abordada, compreendese que a população idosa tem sido alvo de pesquisadores em seus diferentes estudos envolvidos. Embora seja uma temática amplamente discutida, ainda há uma lacuna no conhecimento científico produzido sobre a visão de cuidadores formais de idosos em Instituições de Longa Permanência.

Ademais, informações relativas aos cuidadores formais, como atividade profissional, poderão indicar tendências, deficiências e necessidades relativas a este profissional no mercado de trabalho (LOPES et al., 2012), além de contribuir para nortear políticas públicas específicas para a população de idosos dependentes institucionalizados. Nesta direção, a pergunta de pesquisa que delineou o presente estudo foi: Qual a visão de cuidadores formais no cuidado de idosos dependentes que residem em Instituições de Longa Permanência? Em busca dessa compreensão, o objetivo desse estudo foi analisar a visão de cuidadores formais no cuidado de idosos dependentes institucionalizados.

\section{Métodos}

Trata-se de um estudo descritivo-exploratório, com abordagem qualitativa, selecionada em razão da natureza do objeto focalizado, qual seja: os processos simbólicos da visão de cuidadores no cuidado de idosos dependentes institucionalizados. Cabe ainda assinalar aproximações desse estudo no âmbito da avaliação qualitativa dos serviços prestados a idosos institucionalizados.

O cenário desta pesquisa se configurou em uma Instituição de Longa Permanência para Idosos (ILPI), de caráter filantrópico, sediada na zona leste da área urbana da cidade de Teresina, estado do Piauí, na região nordeste do Brasil. Tendo em vista o objetivo proposto, a escolha desta ILPI aconteceu de forma intencional, em decorrência de sua expressividade no desenvolvimento 
de pesquisas acadêmicas. O consentimento da direção administrativa da ILPI desde o início do delineamento da proposta deste estudo foi decisivo. No momento da realização desta pesquisa, a referida ILPI atendia 35 idosos, sendo 18 na ala masculina e 17 na ala feminina, e contava com a atuação de 13 cuidadores formais.

O número de participantes foi delimitado por saturação teórica, na qual, à medida que os dados eram obtidos e/ou analisados, as estruturas de relevância se aprofundavam, respondendo progressivamente aos objetivos delineados apontando consistência ante as questões em estudo (FONTANELLA et al., 2011). Como critérios de inclusão estabeleceram-se os seguintes: identificar-se como cuidador formal de um idoso dependente, residente na ILPI selecionada para a realização desta pesquisa, há pelo menos um ano, por se acreditar que a maior trajetória do cuidado possibilita informações relevantes a respeito do objeto do estudo delineado e ter idade igual ou superior a 18 anos. Dessa forma, dos 13 cuidadores formais atuantes na ILPI selecionada, dois foram excluídos, pois um encontrava-se de licença saúde e o outro de férias, e dois não concordaram em participar do estudo.

Os dados foram coletados nos meses de dezembro de 2014 a março de 2015, por meio de entrevistas gravadas, utilizando um roteiro temático semiestruturado elaborado pelos próprios pesquisadores do estudo para a coleta das informações, com três questões, a saber:

- Questão 1: Fale sobre como é cuidar do idoso dependente institucionalizado.

- Questão 2: Fale sobre as dificuldades para cuidar de um idoso dependente institucionalizado.

- Questão 3: Fale sobre as facilidades para cuidar de um idoso dependente institucionalizado.

Visando caracterizar os participantes desse estudo, foram coletadas informações sociodemográficas por meio um formulário, contemplando as seguintes variáveis: gênero, procedência, idade, religião, cor/raça, estado civil, escolaridade, renda familiar e ocupação profissional.

As entrevistas foram previamente agendadas e realizadas em uma sala climatizada e iluminada, no local do estudo, somente com a presença do pesquisador, conforme a disponibilidade dos participantes, com tempo médio de duração de 16 minutos. As entrevistas foram gravadas com auxílio de um gravador digital portátil. Os discursos proferidos foram integralmente transcritos, primando-se por manter a veracidade das informações obtidas.

Em seguida, utilizou-se como estratégia metodológica em pesquisa qualitativa a construção do Discurso do Sujeito Coletivo (DSC). A técnica do 
DSC consiste em selecionar, de cada resposta individual a uma questão, as expressões-chave (E-CH), que são trechos mais significativos destas respostas. Às E-CH correspondem ideias centrais (IC), que são a síntese do conteúdo discursivo manifestado nas E-CH. Com o material das E-CH e das IC constroem-se discursos-síntese na primeira pessoa do singular, que são os DSC, onde o pensamento de um grupo ou coletividade aparece como um discurso individual (LEFEVRE, F.; LEFEVRE, A.; MARQUES, 2009).

Ressalta-se que a pesquisa foi realizada obedecendo aos princípios da Resolução no .466 de 12 de dezembro de 2012 do Conselho Nacional de Saúde (BRASIL, 2012a). A pesquisa recebeu aprovação do Comitê de Ética em Pesquisa do Centro Universitário de Saúde, Ciências Humanas e Tecnológicas do Piauí - UNINOVAFAPI, sediado na cidade de Teresina, Piauí, Brasil, sob o CAAE no. 38556214.6.0000.5210 e Parecer no. 888.897/2014, em 27 de novembro de 2014. Como forma de preservar o anonimato dos participantes, optou-se por substituir os nomes dos nove cuidadores por codinomes de estrelas do universo (Aludra, Atria, Capella, Mimosa, Mintaka, Mizar, Sargas, Shaula e Vega), fazendo jus a relevância no cuidado ao idoso institucionalizado dependente.

\section{Resultados e discussão}

Participaram deste estudo nove cuidadores, com prevalência do gênero feminino (78\%), procedentes da cidade de Teresina (89\%). Em relação ao gênero, esse dado corrobora com os apresentados na literatura e, segundo Lima et al. (2016) é justificado pelo fator histórico, pois a mulheres tem sido consideradas, ao longo dos anos, o principal cuidador de sua família e filhos, quer seja no ambiente familiar ou institucional. Quanto a idade dos entrevistados, a média foi 45 anos, idade próxima ao verificado no estudo de Lima et al. (2016), o qual apresenta idade média de 42,6 anos e que vão de encontro a outros estudos em que aproximadamente $40 \%$ da amostra têm mais de 60 anos. Estudo internacional constatou que cerca de um em cada três cuidadores tem 65 anos ou mais, indicando que os idosos estão cuidando de idosos (GAIOLI; FUREGATO; SANTOS, 2012; ALZHEIMER'S ASSOCIATION, 2016).

A religião católica representou $78 \%$ dos entrevistados. A cor/raça parda (67\%) foi a mais a autodeclarada, dados equivalentes ao apresentado por outros dois estudos realizados no Nordeste do Brasil, 74,1\% e 65\% (ANJOS et al., 2015; ANJOS; BOERY; PEREIRA, 2014). No que se refere ao estado civil, a maioria era casado (56\%), sendo comum esse dado a outros dois estudos em que a porcentagem foi de 58,1 e 52\%, sinalizando que, além da tarefa de 
cuidar do idoso, estes possuem outras tarefas e responsabilidades, como os afazeres domésticos e atenção familiar, o que pode implicar em sobrecarga de atividades (ARAUJO et al., 2013; LOPES et al., 2012).

O primeiro grau completo $(44,5 \%)$ foi o nível de escolaridade que prevaleceu entre os cuidadores, tal qual apresentado em um estudo em Ribeirão Preto, em que $58 \%$ dos cuidadores possuíam o ensino fundamental completo e, contrariamente aos dados apresentados em outros estudos em que a população analisada apresenta baixo nível de escolaridade (67,3\%), analfabetos ou não possuíam o ensino fundamental completo (GAIOLI; FUREGATO; SANTOS, 2012; OLIVEIRA et al., 2011). Evidenciando a importância da escolaridade para os cuidadores de idosos, Tabeleão, Tomasi e Quevedo (2014) afirmam que o baixo nível dessa variável pode implicar em dificuldade do cuidador em assimilar conhecimentos e informações sobre a doença, sobre o envelhecimento, fator que a afeta diretamente e consideravelmente sua sobrecarga de trabalho.

Os resultados do discurso do sujeito coletivo foram apresentados em três categorias temáticas: a visão do cuidador do idoso dependente institucionalizado, dificuldades do cuidador para cuidar de um idoso dependente institucionalizado e facilidades do cuidador para cuidar de um idoso dependente institucionalizado.

\subsection{A visão do cuidador do idoso dependente institucionalizado}

Esta primeira categoria foi gerada em conformidade com as respostas dos participantes quando estimulados a responder a primeira questão norteadora realizada na entrevista, "Fale sobre como é cuidar do idoso dependente institucionalizado". Neste contexto, por meio dos discursos dos participantes, três ideias centrais foram elaboradas. Cada ideia central foi codificada pelas letras IC, seguida por uma letra em ordem alfabética sequencial, para diferenciar uma ideia central de outra: IC A - Satisfação no cuidado, IC B - Complexidade do cuidado e IC C - Relacionamento entre o idoso e o cuidador (Tabela 1). 
Tabela 1 - Ideias centrais, discurso do sujeito coletivo, participantes e frequência dos cuidadores formais participantes do estudo da primeira categoria temática "A visão do cuidador do idoso dependente institucionalizado". Teresina/PI, Brasil, 2015.

\begin{tabular}{|c|c|c|}
\hline IC A - Satisfação no cuidado & Participantes & Frequência \\
\hline $\begin{array}{l}\text { "Me satisfaz muito poder cuidar, ajudar as pessoas, dar } \\
\text { atenção, carinho, amor [...] principalmente na fase da } \\
\text { vida em que esses idosos mais precisam". }\end{array}$ & $\begin{array}{l}\text { Aludra, Atria } \\
\text { Mimosa, Mintaka, } \\
\text { Shaula, Vega }\end{array}$ & $6(66,6 \%)$ \\
\hline IC B - Complexidade do cuidado & Participantes & Frequência \\
\hline $\begin{array}{l}\text { "Não é fácil, pois você tem que estar atenta a tudo [...], } \\
\text { tem que saber sobre os remédios, a comida, porque } \\
\text { nem todo mundo come a mesma comida, } \\
\text { é complicado". }\end{array}$ & $\begin{array}{l}\text { Capella, Mizar, } \\
\text { Sargas }\end{array}$ & $3(33,3 \%)$ \\
\hline IC B - Relacionamento entre o idoso e o cuidador & Participantes & Frequência \\
\hline $\begin{array}{l}\text { "Cuido deles como se fossem alguém da minha família. } \\
\text { Já conheço o jeitinho de cada um pela convivência. } \\
\text { Quando eles amanhecem tristes, sem querer conversar, } \\
\text { percebo que essa tristeza é por estar longe dos seus } \\
\text { familiares, então eu converso com eles e passa". }\end{array}$ & $\begin{array}{l}\text { Aludra, Atria, } \\
\text { Capella, Mimosa, } \\
\text { Mintaka, Shaula, } \\
\text { Vega }\end{array}$ & $7(77,7 \%)$ \\
\hline
\end{tabular}

Fonte: Tabela elaborada pelos autores.

Observa-se pelos trechos dos discursos apresentados na IC A que o sentimento de satisfação no cuidado dos cuidadores para os idosos dependentes institucionalizados esteve presente com frequência de 66,6\%. De acordo com o achado da pesquisa de Pinto e Oliveira (2015), as relações sociais dos idosos são fundamentais para a manutenção da qualidade de vida no envelhecimento. Segundo Scalco et al. (2013), a história do relacionamento entre o cuidador e o idoso mostra-se bastante importante para compreender não só o surgimento, mas a construção dos sentimentos e da subjetividade do cuidador, na medida em que ela se constrói na relação com a pessoa cuidada. "Dar atenção, carinho, amor" é a satisfação que o cuidador tem quando o retorno do bom relacionamento é apresentado em gratificação por parte do idoso, portanto, o cuidado humanizado se efetiva na descrição das entrevistas. Dessa forma, os cuidadores do estudo se valeram dessa construção de sentimentos, em busca de proporcionar uma convivência mais agradável, afetuosa e condizente com a proposta de cuidar. Entretanto, verificou-se conforme a IC B que cuidar de um idoso dependente institucionalizado pode ser complexo, especialmente devido à execução da rotina de cuidados diários, principalmente medicações e alimentação. De acordo com Ballarin 
et al. (2016), fatores como a gravidade da doença, o comprometimento na habilidade de comunicação e o nível de incapacidade funcional do idoso estão associados ao nível de sobrecarga de trabalho e a qualidade de vida do cuidador formal, pois os medicamentos apresentam horários e a alimentação é uma rotina diária específica para cada idoso. No tocante às especificidades, para Patrocinio (2015), é de fundamental importância que o cuidador formal esteja capacitado para isto: para conhecer as medicações, seus horários, seus efeitos e a alimentação adequada a cada cliente.

Nos trechos da IC C, foi possível perceber a relação familiar desenvolvida entre o cuidador e o idoso dependente. Na visão do cuidador, a falta da vínculo familiar do idoso dependente é maléfico e, sabendo disso, tenta suprir essa necessidade. O estudo de Camargo (2010) ressalta a relevância do bom relacionamento afetivo construído entre o cuidador e o idoso como um vínculo familiar, mostrando-se muito importante para compreender a construção dos sentimentos e da subjetividade do cuidador, construída durante a relação com o idoso (SCALCO et al., 2013).

\subsection{Dificuldades do cuidador para cuidar de um idoso dependente institucionalizado}

A segunda categoria foi gerada a partir das respostas dos cuidadores quando estimulados a responder a segunda questão norteadora realizada na entrevista, "Fale sobre as dificuldades para cuidar de um idoso dependente institucionalizado". Assim, por meio dos discursos dos participantes, três ideias centrais foram elaboradas: IC A - Rotina de cuidados, IC B - Fragilidades físicas e emocionais e IC C - Deambulação noturna (Tabela 2). 
Tabela 2 - Ideias centrais, discurso do sujeito coletivo, participantes e frequência dos cuidadores formais participantes do estudo da segunda categoria temática "Díficuldades do cuidador para cuidar de um idoso dependente institucionalizado". Teresina/PI, Brasil, 2015.

\begin{tabular}{|c|c|c|}
\hline IC A - Rotina de cuidados & Participantes & Frequência \\
\hline $\begin{array}{l}\text { "Todos os idosos têm que ter hora certa para banhar, } \\
\text { comer, dormir, tomar seus remédios. Tudo isso mudou um } \\
\text { pouco a minha rotina, pois eu nunca tinha trabalhado em } \\
\text { um abrigo que cuida de idoso, ainda mais dependente". }\end{array}$ & $\begin{array}{l}\text { Aludra, Atria, } \\
\text { Capella, Mimosa, } \\
\text { Mintaka, Mizar, Sar- } \\
\text { gas, Shaula, Vega }\end{array}$ & $9(100 \%)$ \\
\hline IC B - Fragilidades físicas e emocionais & Participantes & Frequência \\
\hline $\begin{array}{l}\text { "Por causa da idade, eles são mais frágeis, e o cuidado aqui } \\
\text { tem que ser redobrado, porque se você perder a atenção } \\
\text { um minuto qualquer, eles podem cair, e o problema fica } \\
\text { maior, a atenção tem que ser } 24 \text { horas por dia". "quando } \\
\text { eles ficam zangados é muito difícil, pois eles mudam } \\
\text { totalmente e a gente tem que ficar bajulando, pois eles não } \\
\text { querem fazer nada". }\end{array}$ & $\begin{array}{l}\text { Aludra, Capella, } \\
\text { Mizar, Mintaka, } \\
\text { Mimosa, Sargas } \\
\text { Vega }\end{array}$ & $7(77,7 \%)$ \\
\hline IC B - Deambulação noturna & Participantes & Frequência \\
\hline $\begin{array}{l}\text { "Alguns aqui têm muita insônia, dormem muito durante o dia } \\
\text { e, quando dá de madrugada, querem ficar caminhando, por } \\
\text { isso durante a noite, tem que sair dando umas voltas nos } \\
\text { quartos e prestando atenção em tudo". }\end{array}$ & $\begin{array}{l}\text { Aludra, Atria, } \\
\text { Mimosa, Mintaka, } \\
\text { Shaula, Vega }\end{array}$ & $6(66,6 \%)$ \\
\hline
\end{tabular}

Fonte: Tabela elaborada pelos autores.

Observa-se na Tabela 2 que todos cuidadores entrevistados (100\%) relataram dificuldades em relação à adaptação com os horários de rotina dos cuidados prestados (IC A). Dessa forma, o estudo de Alves-Silva, Scorsolini-Comin e Santos (2013) destacou que as ILPI apresentam um forte poder disciplinar e são marcadas por regras rígidas e rotina regida por horários determinados para as atividades diárias, como o banho, a alimentação, os medicamentos e o descanso. Assim, o estudo de Oliveira et al. (2015) enfatiza que cuidar e ser cuidado, especialmente em situação de dependência, pode ser uma condição estressante para ambos os atores desta dinâmica relacional. Percebe-se que as tarefas dos cuidadores, mediadas pelas repetidas atividades diárias rotineiras, na maioria das vezes, podem gerar sobrecarga, podendo interferir no seu cotidiano pessoal.

Outra dificuldade apresentada nos relatos diz respeito às fragilidades físicas e emocionais (IC B) dos idosos relatadas pelos cuidadores, dentre as quais podem ser destacadas as alterações de humor, apresentando-se como idosos zangados ou irritados. Por esta ideia, o estudo de Rezende, Lodovici e 
Concone (2012) mostrou uma visão negativa de ser idoso, relacionando-o com dependência, incapacidade, tristeza, depressão e mau-humor. Entretanto, outro estudo concluiu que o fato de alguns idosos se apresentarem descontentes e irritados com a situação de ser idoso pode estar associado à institucionalização contra a sua vontade e/ou por estar longe da família (OLIVEIRA et al., 2015). Assim, pode-se inferir que os cuidadores formais de idosos em ILPI devem manter o bom-humor e um ambiente com alegria, podendo auxiliar tanto o próprio cuidador no decorrer da execução das atividades de rotina como também no alívio da tensão e o estado de humor dos idosos institucionalizados.

No que concerne à deambulação noturna dos idosos, conforme observado nos discursos da IC C, há uma preocupação por parte do cuidador na ocorrência de quedas. Essa preocupação não é em vão. Os dados apresentados na literatura fundamentam essa inquietação, pois a ocorrência de quedas é bastante comum nos idosos, devido as próprias alterações decorrentes do processo de senescência e pelo aparecimento de patologias, além dos fatores do próprio ambiente no qual o idoso está introduzido (REIS; ROCHA; DUARTE, 2014). Pesquisas mostram que a maioria das quedas ocorre predominantemente no período diurno (BARBOSA et al., 2014; SANTOS; WEIZENMANN; KOETZ, 2015). Entretanto, as quedas que ocorrem no período noturno podem estar relacionadas ao deslocamento dos idosos para beber água ou para ir ao banheiro e, muitas vezes, deparando-se com condições ambientais desfavoráveis, como a iluminação inadequada.

A apreensão do cuidador formal sobre as quedas é mais orientada quanto maior foi o tempo de convivência com o idoso, pois o conhecimento do cuidador sobre a rotina do ser cuidado é aumentada. Portanto, a ocorrência de quedas pode ser prevenida (ALVES-SILVA; SCORSOLINI-COMIN; SANTOS, 2013). Sugere-se que os fatores de risco sejam minimizados.

\subsection{Facilidades do cuidador para cuidar de um idoso dependente institucionalizado}

Por fim, a terceira categoria gerada diz respeito às análises dos discursos dos cuidadores quando estimulados a responder a terceira questão norteadora realizada na entrevista, "Fale sobre as facilidades para cuidar de um idoso dependente institucionalizado". Desta forma, duas ideias centrais foram elaboradas. Cada ideia central foi codificada pelas letras IC, seguida por uma letra em ordem alfabética sequencial, para diferenciar uma ideia central de outra. As duas ideias centrais reveladas nesta categoria foram: IC A - Bom-humor e IC B - Comunicação (Tabela 3). 
Tabela 3 - Ideias centrais, discurso do sujeito coletivo, participantes e frequência dos cuidadores formais participantes do estudo da terceira categoria temática "Facilidades do cuidador para cuidar de um idoso dependente institucionalizado". Teresina/PI, Brasil, 2015.

\begin{tabular}{l|l|c}
\hline IC A - Bom-humor & \multicolumn{1}{|c}{ Participantes } & Frequência \\
\hline $\begin{array}{l}\text { "Quando eles estão de bom humor, a troca que a } \\
\text { gente consegue ter com eles é muito significativa". "aa } \\
\text { rotina costuma ser mais agradável quando eles estão } \\
\text { sorridentes... isso ajuda muito a gente que cuida". }\end{array}$ & $\begin{array}{l}\text { Aludra, Atria } \\
\text { Capella, Mimosa, } \\
\text { Mizar, Sargas, Shaula, } \\
\text { Vega }\end{array}$ & 8 (88,8\%) \\
\hline IC B - Comunicação & \multicolumn{1}{|c}{ Participantes } & Frequência \\
\hline $\begin{array}{l}\text { "As facilidades? São muitas, porque o amor que tenho } \\
\text { por eles é enorme, a gente conversa, trocamos muitas } \\
\text { ideias, eles são minha segunda família e o amor que } \\
\text { você passa a sentir por eles é muito grande". "É muito } \\
\text { gratificante tudo isso, principalmente quando eles } \\
\text { demonstram o amor quizar, }\end{array}$ & Megar, Shaula damos...". & $7(77,7 \%)$ \\
\hline
\end{tabular}

Fonte: Tabela elaborada pelos autores.

Conforme pode ser visualizado da Tabela 3, uma das facilidades relatada pelos cuidadores durante as entrevistas foi o bom-humor dos idosos, o estar sorridente. Assim, buscando embasamentos no estudo de Paschoalin e Perensim (2015), pode-se afirmar que é mais fácil manter um bom relacionamento com os idosos, uma aproximação ou uma troca, pois são pessoas bem-humoradas, receptivas e comunicativas. Nesse sentido, a síntese dos achados de artigos científicos de uma revisão integrativa permitiu que os pesquisadores concluíssem que além da competência técnica e a habilidade pessoal durante o cuidado com a pessoa idosa em uma ILPI, a comunicação interpessoal é um fator determinante (DIAS et al., 2014). Conforme os relatos destacados na Tabela 3 observa-se que os cuidadores se revelaram satisfeitos com a qualidade da comunicação que possuíam entre os idosos institucionalizados, sendo importante, na visão deles, para redução de barreiras que comprometem o relacionamento e os cuidados oferecidos.

Ainda nos discursos observados na Tabela 3, observa-se que o amor e a aproximação afetuosa entre o cuidador e o idoso também foi uma das facilidades encontrada nos relatos. Entretanto, torna-se interessante destacar que as facilidades para o cuidado com a pessoa idosa não devem depender unicamente do idoso, do seu humor, da sua personalidade e do seu estado; elas podem ser proporcionadas pelo próprio cuidador, na medida em que ele busca alternativas para tornar fácil o contato e a aproximação (PASCHOALIN; PERENSIM, 2015). 
Neste sentido, o estudo português desenvolvido por Carvalho (2014) revelou muitos sentimentos positivos mencionados pelos cuidadores no cuidado com idosos institucionalizados. Segundo o estudo, os cuidadores sentiram orgulhosos por desempenharem o papel de cuidar, devido ao reconhecimento da importância que os idosos revelaram a respeito dos cuidadores, os quais manifestaram o sentimento de dever cumprido. Ao vislumbrar todo esse contexto, pode-se inferir a partir desses resultados que o bem-estar emocional do idoso, representado pelo bom-humor na IC A da Tabela 3, torna-se muito relevante para facilitar a execução do cuidado pelo cuidador.

\section{Considerações finais}

Este estudo evidenciou que o ato de cuidar de idosos dependentes, na visão do cuidador, ultrapassa o próprio aspecto da rotina diária e do relacionamento afetivo, repercutindo na atenção integral, holística e humanizada praticada pelo cuidador sobre a melhora na qualidade de vida de idosos dependentes institucionalizados. A visão do cuidador formal abrangeu aspectos de satisfação, complexidade no cuidado e relacionamentos entre o cuidador e o idoso. Para tanto, esse cuidador desvelou as mais profundas vivências para apresentar a construção dos seus relatos, demostrando a relevância intrínseca que esse cuidador tem para com as atividades de atenção quer sejam elas favoráveis, quer sejam elas desvantajosas ao bom desempenho do cuidado.

No tocante às desvantagens, muitas delas estavam relacionadas à rotina de cuidado ao idoso dependente, possibilitando evidenciar as dificuldades dos cuidadores entrevistados nesse estudo foram além dos aspectos físicos dos idosos e vão especialmente ao encontro dos aspectos emocionais, mentais, sociais e até mesmo culturais. Por conseguinte, evidencia-se que as atividades desvantajosas apresentadas na visão dos cuidadores são, de alguma forma, contornada pelas atividades favoráveis do cuidado e com a ajuda de outrem.

Em se tratando dos aspectos facilitadores na rotina de cuidado do idoso dependente, foi possível constatar duas ideias centrais: o bom-humor e a comunicação. Também estiveram presentes nos discursos a valorização do amor e da aproximação afetuosa entre o cuidador e o idoso, facilitando o cuidado da rotina diária. Os seus conhecimentos, suas vivências representam o impulso para a ampliação da visão de cuidado e assim entender, também, que precisam de atividades de promoção do seu bem-estar promovidas pelas ILP-s com o intuito de favorecer um bom estado emocional e desenvolver estratégias de 
prevenção contra as dificuldades ocasionadas pelas atividades inerentes à rotina de cuidados.

Dessa forma, espera-se que a contribuição dos relatos dos cuidadores nesse estudo possibilite um maior conhecimento e discussão sobre a temática, facilitando a compreensão, a resolução e a utilização de estratégias de cuidados por parte dos cuidadores formais. Assim, os cuidadores podem implementar, em suas práticas, um cuidado pautado na redução de dificuldades e desenvolvimento de facilidades para que as mesmas sejam aliadas à rotina diária do cuidado.

Como fator limitante deste estudo, destaca-se a carência de publicações que envolvam a visão de cuidadores formais em ILPI, dificultando as comparações com outros estudos da mesma natureza durante a discussão dos resultados. Ressalta-se também que, tendo em vista a abordagem qualitativa selecionada, a coleta dos dados dessa pesquisa foi desenvolvida em uma única realidade específica, situada na região Nordeste do Brasil. Dessa forma, recomenda-se que esse desenho de estudo seja reproduzido em outras ILPI locais, demais regiões brasileiras e outros países com culturas diferentes, a fim de ampliar a compreensão do cuidado ao idoso dependente institucionalizado.

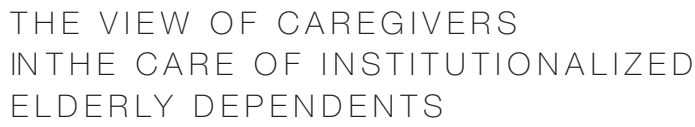

abstract

This study aims to analyze the view of formal caregivers in the care of institutionalized elderly dependents. We conducted a qualitative study, developed in a Long Term Care Institutions for Elders in Teresina, Piaui, Brazil. They attended nine caregivers that provided care with institutionalized elderly dependents. Data were collected from December/2014 to March/2015, through interviews, using a semi-structured thematic guide. The recorded interviews were transcribed and analyzed by speech technique of collective subject. The results were presented in three thematic categories and three tables containing the central ideas, the selected passages of individual speeches, participants and frequency. In the first category, the caregiver's view of the institutionalized dependent elderly, were found three central ideas: satisfaction in care, complexity of care and relationship between the elderly and the caregiver. In the second category, caregiver difficulties to take care of an institutionalized elderly dependent were also 
generated three central ideas: routine care, physical and emotional weaknesses and night walking. Finally, the third category, caregiver facilities to care for an institutionalized dependent elderly, were found two central ideas: good humor and communication. We recommend that the Long Term Care Institutions for Elders pay greater attention to activities that promote well-being of caregivers, as well as prevention strategies against the difficulties caused by the activities related to routine care for institutionalized elderly dependents.

keywords

Caregivers. Aged. Frail elderly.

referências

ALVES-SILVA, Júnia Denise; SCORSOLINI-COMIN, Fabio; SANTOS, Manoel Antônio dos. Idosos em instituições de longa permanência: desenvolvimento, condições de vida e saúde. Psicologia: reflexão e crítica, Porto Alegre, v. 26, n. 4, p. 820-830, dez. 2013.

ALZHEIMER'S ASSOCIATION. 2016 Alzheimer's disease facts and figures. Alzheimer's and Dementia, v. 12, n. 4, p. 459-509, abr. 2016.

ANJOS, Karla Ferraz dos; BOERY, Rita Narriman Silva de Oliveira; PEREIRA, Rafael. Quality of life of relative caregivers of elderly dependents at home. Texto \& Contexto Enfermagem, Florianópolis, v. 23, n. 3, p. 600-608, set. 2014.

ANJOS, Karla Ferraz dos et al. Associação entre apoio social e qualidade de vida de cuidadores familiares de idosos dependentes. Ciência \& Saúde Coletiva, Rio de Janeiro, v. 20, n. 5, p. 1321-1330, maio 2015.

ANJOS, Karla Ferraz dos et al. Perfil de cuidadores familiares de idosos no domicílio. Revista de Pesquisa: cuidado é fundamental, Rio de Janeiro, v. 6, n. 2, p. 450-461, abr./jun. 2014.

ARAUJO, Jeferson Santos et al. Profile of caregivers and difficulties in elderly care in the city of Ananindeua, State of Para, Brazil. Revista Brasileira de Geriatria e Gerontologia, Rio de Janeiro, v. 16, n. 1, p. 149-158, jul. 2013.

BALLARIN, Maria Luisa Gazabim Simões et al. Perfl sociodemográfco e sobrecarga de cuidadores informais de pacientes assistidos em ambulatório de terapia ocupacional. Cadernos Brasileiros de Terapia Ocupacional, São Carlos, v. 24, n. 2, p. 315-321, dez./out. 2016.

BARBOSA, Keylla Talitha Fernandes et al. Caracterização das quedas referidas por idosos. Revista Baiana de Enfermagem, Salvador, v. 28, n. 2, p. 168-175, mai/ago. 2014.

BRASIL. Ministério da Saúde. Conselho Nacional de Saúde. Comissão de ética e Pesquisa (CONEP). Resolução n 466, de 12 de dezembro de 2012. Diário Oficial [da] República Federativa do Brasil, Brasilia, DF, 2012a. Disponível em: <http://conselho. saude.gov.br/resolucoes/2012/Reso466.pdf>. Acesso em: 14 jul. 2016.

Câmara dos Deputados. Projeto de Lei n 4702, de 09 de novembro de 2012. Dispõe sobre o exercício da profissão de cuidador de pessoa idosa e dá outras providências. Diário Oficial [da] República Federativa do Brasil, Brasília, DF, 2012b. 

oposicao $=559429$ >. Acesso em: 12 jul. 2016.

CAMARGO, Renata Cristina Virgolin Ferreira de. Implicações na saúde mental de cuidadores de idosos: uma necessidade urgente de apoio formal. SMAD: revista eletrônica saúde mental álcool e drogas, Ribeirão Preto, v. 6, n. 2, ago. 2010.

CARVALHO, Matilde Sampaio. O cuidado paliativo a idosos institucionalizados: vivência dos cuidadores. 2014. 250f. Dissertação (Mestrado em Cuidados Paliativos)-Faculdade de Medicina do Porto, Porto, 2014.

DIAS, Kalina Coeli Costa de Oliveira et al. O cuidado em enfermagem direcionado para a pessoa idosa: revisão integrativa. Revista de Enfermagem UFPE, Recife, v. 8, n. 5, p. 1337-1346, mai. 2014.

FONTANELLA, Bruno Jose Barcellos et al. Amostragem em pesquisas qualitativas: proposta de procedimentos para constatar saturação teórica. Cadernos de Saúde Pública, Rio de Janeiro, v. 27, n. 2, p. 389-394, fev. 2011.

GAIOLI, Cheila Cristina Leonardo de Oliveira; FUREGATO, Antonia Regina Ferreira; SANTOS, Jair Lício Ferreira. Perfil de cuidadores de idosos com doença de Alzheimer associado à resiliência. Texto \& Contexto Enfermagem, Florianópolis, v. 21, n. 1, p. 150-157, mar. 2012

INSTITUTO BRASILEIRO DE GEOGRAFIA EESTATÍSTICA (IBGE). Síntese de indicadores sociais: uma análise das condições de vida da população brasileira. Rio de Janeiro: IBGE, 2014. (Série Estudos e Pesquisas: informação demográfica e socioeconômica, 34). Disponível em: <http://biblioteca.ibge.gov.br/visualizacao/livros/liv91983.pdf>. Acesso em: 15 jul. 2016.

LEFEVRE, Fernando: LEFEVRE, Ana Maria Cavalcanti; MARQUES, Maria Cristina da Costa. Discurso do Sujeito Coletivo: complexidade e auto-organização. Ciência \& Saúde Coletiva, Rio de Janeiro, v. 14, n. 4, p. 1193-1204, jul. 2009.

LIMA, Raquel Janyne et al. Profle of Caregivers of Institutionalized Elders. International Archives of Medicine, London, v. 9, n. 131, p. 1-8, jul. 2016.

LINO, Valéria Teresa Saraiva et al. Prevalência de sobrecarga e respectivos fatores associados em cuidadores de idosos dependentes, em uma região pobre do Rio de Janeiro, Brasil. Cadernos de Saúde Pública, Rio de Janeiro, v. 32, n. 6, jun. 2016.

LISBOA Cristiane Rabelo; CHIANCA, Tânia Couto Machado. Perfil epidemiológico, clínico e de independência funcional de uma população idosa institucionalizada. Revista Brasileira de Enfermagem, Brasilia, DF, v. 65, n. 3, p. 574-579, mai./jun. 2012.

LOPES, Renate Antunes et al. Perfil dos cuidadores das instituições de longa permanência para idosos de Itaúna - MG. ConScientiae Saúde, São Paulo, v. 11, n. 2, p. 338-344, abr./jun. 2012.

LOREIRO, Lara de Sá Neves et al. Sobrecarga de cuidadores familiares de idosos: prevalência e associação com características do idoso e do cuidador. Revista da Escola de Enfermagem da USP, São Paulo, v. 47, n. 5, p. 1133-1140, 2013.

MORALES-VIVES, Fabia; VIGIL-COLET, Andreu. Are old people so gentle?: functional and dysfunctional impulsivity in the elderly. International Psychogeriatrics, Melbourne, v. 24, n. 3, p. 465-471, mar. 2012

OLIVEIRA, Déborah Cristina de et al. Qualidade de vida e sobrecarga de trabalho em cuidadores de idosos em seguimento ambulatorial. Texto \& Contexto Enfermagem, Santa Catarina, v. 20, n. 2, p. 234-40, abr./jun. 2011.

OLIVEIRA, Joice Mara de et al. Alterações físico-sociais decorrentes do envelhecimento na perspectiva de idosos institucionalizados. Revista Kairós, São Paulo, v. 18, n. 4, p. 197-213, out./dez. 2015 
ORGANIZAÇÃO DAS NAÇÕES UNIDAS (ONU). World population prospects: the 2010 revision. New York: UN, 2012. Disponivel em: <http://esa.un.org/wpp/P-WPP/htm/ PWPP_Population-Age_65Plus.htm>. Acesso em: 4 nov. 2015.

World population prospects: the 2008 revision. New York: UN, 2009. Disponível em: <http://www.un.org/esa/population/publications/wpp2008/wpp2008_text_tables. pdf>. Acesso em: 4 nov. 2015.

PASCHOALIN, Heloísa Campos; PERENSIM, Katia. A importância da comunicação e do estímulo para a qualidade de vida do idoso. Revista de Enfermagem da UFJF, Juiz de Fora, v. 1, n. 1, p. 45-53, jan/jun. 2015.

PATROCINIO, Wanda Pereira. Autocuidado do cuidador e o cuidado de idosos. Revista Kairós Gerontologia, São Paulo, v. 18, n. 19, p. 99-113, jun. 2015.

PINTO, Francine Náthalie Ferraresi Rodrigues; OLIVEIRA, Dayane Capra. Capacidade funcional e envolvimento social em idosos: há relação? Revista Brasileira de Ciências do Envelhecimento Humano, Passo Fundo, v. 12, n. 1, p. 56-68, jan./abr. 2015.

REIS, Luciana de Araújo; ROCHA, Thais de Souza; DUARTE, Stênio Fernando Pimentel. Quedas: risco e fatores associados em idosos institucionalizados. Revista Baiana de Enfermagem, Salvador, v. 28, n. 3, p. 225-234, set./dez. 2014.

REZENDE, Eliane Garcia; LODOVICl, Flamínia Manzano Moreira; CONCONE, Maria Helena Villas Bôas. A infinitude na religião: quando uma vida só não basta. Revista Kairós: gerontologia, São Paulo, v. 15, n. 4, p. 48-65, ago. 2012.

RISSARDO, Leidyani Karina et al. Sentimentos de residir em uma instituição de longa permanência: percepção de idosos asilados. Revista Enfermagem Uerj, Rio de Janeiro, v. 20, n. 3, p. 380-385, jul./set. 2012.

SANTOS, Marilucia Vieira dos; WEIZENMANN, Simone Ely; KOETZ, Lydia Christmann Espindola. Avaliação dos idosos e a percepção dos profissionais quanto os riscos de quedas em uma instituição de longa permanência. Cinergis, Santa Cruz do Sul, v. 16, n. 1, p. 9-14, jan./mar. 2015

SARAIVA. Dulce Maria Ferraz. O olhar dos e pelos cuidadores: os impactos de cuidar e a importância do apoio ao cuidador. 2011. 135f. Dissertação (Mestrado em Intervenção Social, Inovação e Empreendedorismo)-Faculdade de Psicologia e Ciências da Educação, Porto, 2011.

SCALCO, Janaina Cristina et al. O dia a dia de cuidadores familiares de idosos dependentes. Revista Kairós: gerontologia, São Paulo, v. 16, n. 1, p. 191-208, mar. 2013.

SILVA, Irma Lúcia Silveira et al. Formação profissional de cuidadores de idosos atuantes em instituições de longa permanência. HOLOS, Natal, v. 8, p. 342-356, 2015.

TABELEÃO, Viviane Porto; TOMASI, Elaine; QUEVEDO, Luciana de Ávila. Sobrecarga de familiares de pessoas com transtorno psíquico: níveis e fatores associados. Archives of Clinical Psychiatry, São Paulo, v. 41, n. 3, p. 63-66, fev. 2014.

WANG, J.; ZHAO, X. Family functioning and social support for older patients with depression in an urban area of Shanghai, China. Archives of Gerontology and Geriatrics, Amsterdam, v.55, n.3, p. 574- 579, nov./dez. 2012.

Data de submissão: 04/11/2015

Data de aprovação: 07/04/2017 